\section{Temperature dependent polarity inversion in double-metal terahertz emitters}

\author{
P. C. Gow, D. McBryde, S. A. Berry, and V. Apostolopoulos
}

The temperature dependent polarity inversion in double-metal $\mathrm{THz}$ emitters was investigated. Double-metal emitters utilising different metal pairings and single edge metal emitters were cooled in a helium flow cryostat and their $\mathrm{THz}$ emission was measured over a range of temperatures. Most emitters, including those with insulating layers between the metal and semiconductor, exhibit a flip in polarity of their $\mathrm{THz}$ emission between 50 and $100 \mathrm{~K}$. This shows the inversion is a trait intrinsic to the semiconductor and not influenced by the metallic contact on the surface.

Introduction: Double metal (DM) bias-free terahertz (THz) emitters utilise a combination of the photo-Dember (PD) effect and Schottky currents to generate a net lateral current [1,2]. This net current produces observable THz emission parallel to the direction of the IR pump beam used to excite the emitters. Previous work by the authors explored the temperature characteristics of these emitters [3]. A polarity flip of the emitted $\mathrm{THz}$ radiation was observed for double metal emitters between $80 \mathrm{~K}$ and $120 \mathrm{~K}$. Polarity flips in SI-GaAs have previously been observed to demonstrate a dependence on the polarisation of the exciting beam due to an enhanced electric field at the $\mathrm{Au} / \mathrm{GaAs}$ interface [4]. The flip in polarity was attributed to competing $\mathrm{THz}$ emission mechanisms such as Schottky emission. Fluence dependent polarity inversion has also previously been observed at Au-GaAs interfaces, though without satisfactory explanation [5]. In this letter we explore further the temperature dependent polarity flip demonstrated by DM emitters. We compare the temperature characteristics of a single metal edge emitter with both a standard DM device and one that has a layer between the metals and semiconductor to insulate against Schottky effects, and show that the polarity flip is intrinsic to the semiconductor.

Experiments and results: The emitters investigated were; DM emitters with $\mathrm{Au} / \mathrm{Pb}, \mathrm{Au} / \mathrm{Al}$ and $\mathrm{Cu} / \mathrm{Cr}$ metal pairings and a $\mathrm{DM}$ emitter with $\mathrm{Au} / \mathrm{Pb}$ on top of an insulating $\mathrm{SiO}_{2}$ layer. We have also used single edge metal-semiconductor lateral PD emitters individually fabricated with each of the metals. The different DM emitters and the THz timedomain spectrometer (TDS) setup used in these experiments are described in detail in [3] with the setup shown here in figure 1 for ease. The insulated emitter was fabricated with a $150 \mathrm{~nm}$ thick layer of $\mathrm{SiO}_{2}$ between the $\mathrm{Au} / \mathrm{Pb}$ and the semiconductor, to insulate against band bending from Schottky contacts.

TDS scans showing the polarity dependence for the uninsulated $\mathrm{Au} / \mathrm{Pb}$ emitter are shown in figure 2(a) and the same for the insulated $\mathrm{Au} / \mathrm{Pb}$ emitter is shown in figure 3(a). For the uninsulated emitter the main pulse peak is shifted by $500 \mathrm{fs}$ between $200 \mathrm{~K}$ and $100 \mathrm{~K}$.

Comparing figure 2 (b) and figure 3 (b) directly we see that an increase in signal amplitude occurs at 2 ps for the temperature range of $50-100 \mathrm{~K}$ without being shifted in time. This rise in output is attributed to the

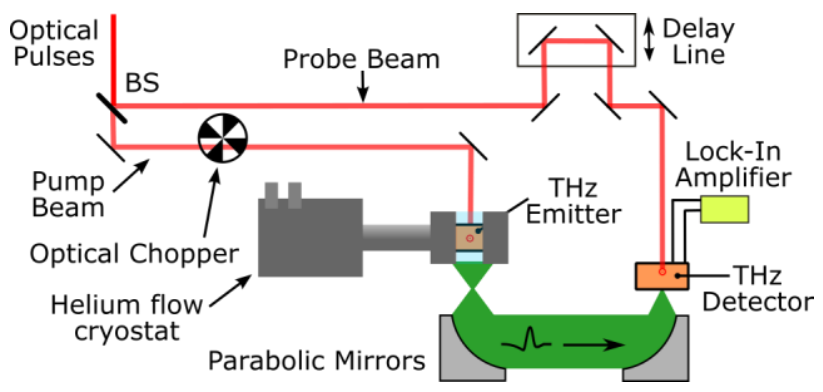

Fig. 1 Diagram of the THz-TDS setup used to measure the temperature dependence of the emitters. Emitters are mounted within a helium flow cryostat and illuminated with a modulated infrared pump beam to excite carriers. A photoconductive detector and lock-in amplifier are used to detect the transient $\mathrm{THz}$ pulse produced. increased photo-Dember efficiency due to carrier mobility as it is evident in both the uninsulated and insulated emitters [6].

The polarity flip is evident both in the uninsulated and insulated $\mathrm{Au} / \mathrm{Pb}$ emitters as well as the $\mathrm{Cu} / \mathrm{Cr}$ emitter. Several tests were carried out to identify contributing factors to the polarity inversion. The polarisation of the pump beam to be parallel or perpendicular to the metal edges, the fluence upon the emitters and the wavelength of the laser was varied between 750 and $850 \mathrm{~nm}$, however, the polarity flip remained evident and unchanged in all cases.

Only the Au/Al DM emitter did not exhibit a polarity flip with temperature. This may be due to the similar skin depths of $\mathrm{Au}$ and $\mathrm{Al}$, meaning emission would be dominated strongly by the metalsemiconductor Schottky fields and not diffusion. At low temperatures any increase in diffusion would be negligible due to the equal-andopposite nature of the dipoles formed under the $\mathrm{Au}$ and $\mathrm{Al}$ edges due to their similar skin depths.

Lateral PD emitters were fabricated with single edges of each metal $(\mathrm{Au}, \mathrm{Pb}, \mathrm{Cu}, \mathrm{Cr}, \mathrm{Al})$ and were mounted within the cryostat to measure the emission of each with temperature. Figure 4 shows the resulting TDS measurements at 4,60 and $240 \mathrm{~K}$ for the single edge Au emitter. The polarity reversal was evident for every single edge emitter utilising only one metal. This suggests that the flip in polarity is linked to some intrinsic property of the SI-GaAs semiconductor competing with $\mathrm{THz}$ emission. Nakajima et al. found evidence for polarity reversal with temperature in bare InP surfaces [7]. This was attributed to competition between the drift current caused by the surface electric field and diffusion currents due to the photo-Dember effect, where at low temperatures the diffusion dominates causing a flip in signal polarity. This is applicable in the case of the single edge emitters but not
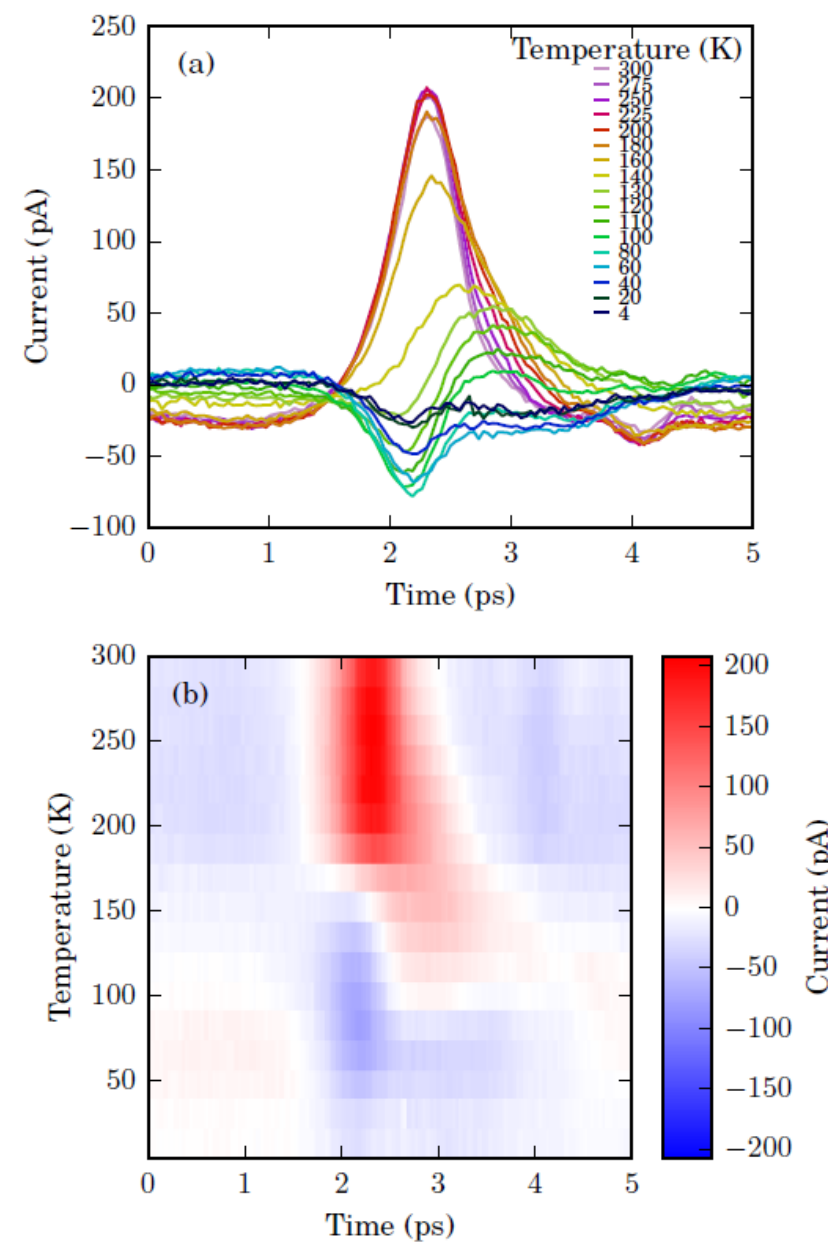

Fig. 2 Temperature dependence THz-TDS scans of an $\mathrm{Au} / \mathrm{Pb} \mathrm{DM}$ emitter fabricated with no insulating layer ranging from 4-300 K. Figure (a) shows the individual THz-TDS of detected current with time superimposed with each other over the range of temperature. Figure (b) shows the same data as figure (a) as a two dimensional image of detected current against temperature and time. 

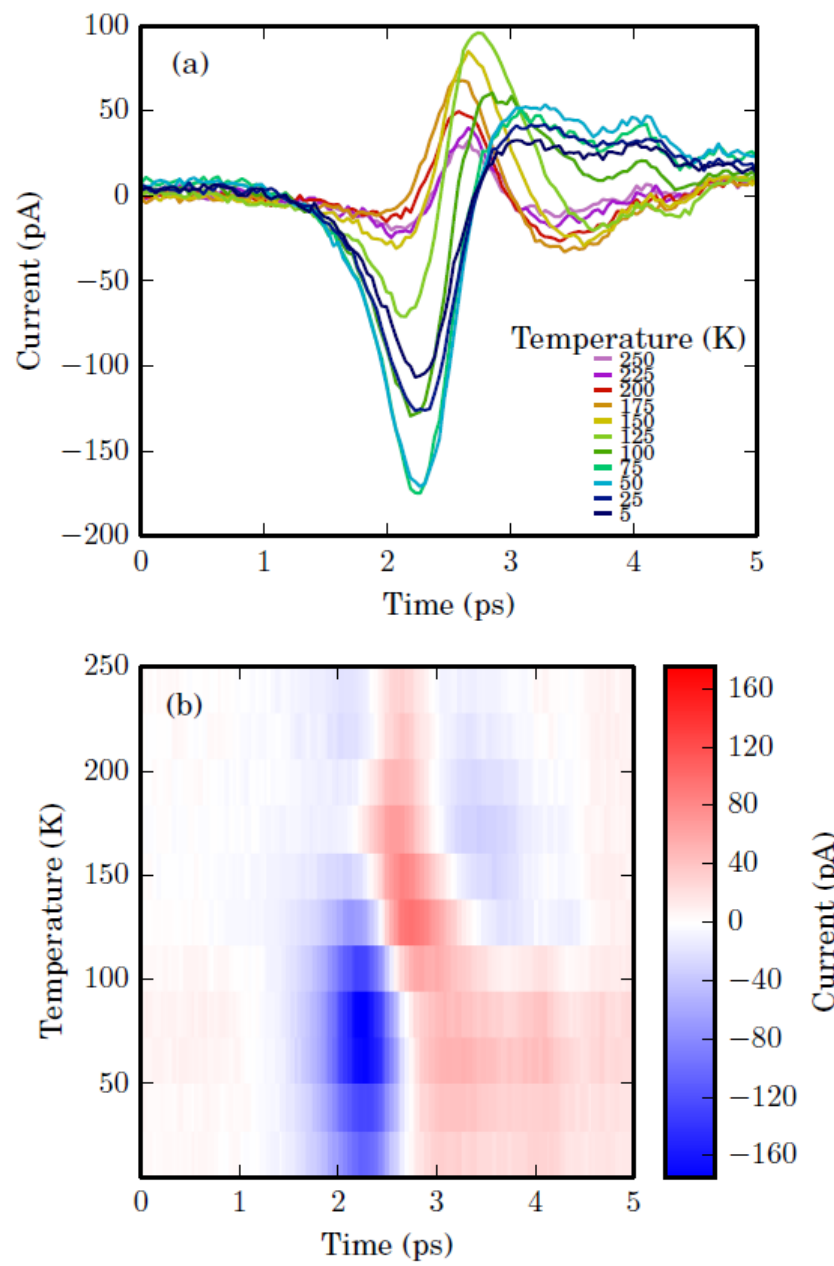

Fig. 3 Temperature dependence THz-TDS scans of an $A u / P b D M$ emitter fabricated with a $\mathrm{SiO} 2$ insulating layer ranging from 5-250 K. Figure (a) shows the individual THz-TDS of detected current with time over the range of temperature. Figure (b) shows the same data as figure (a) as a two dimensional image of detected current against temperature and time.

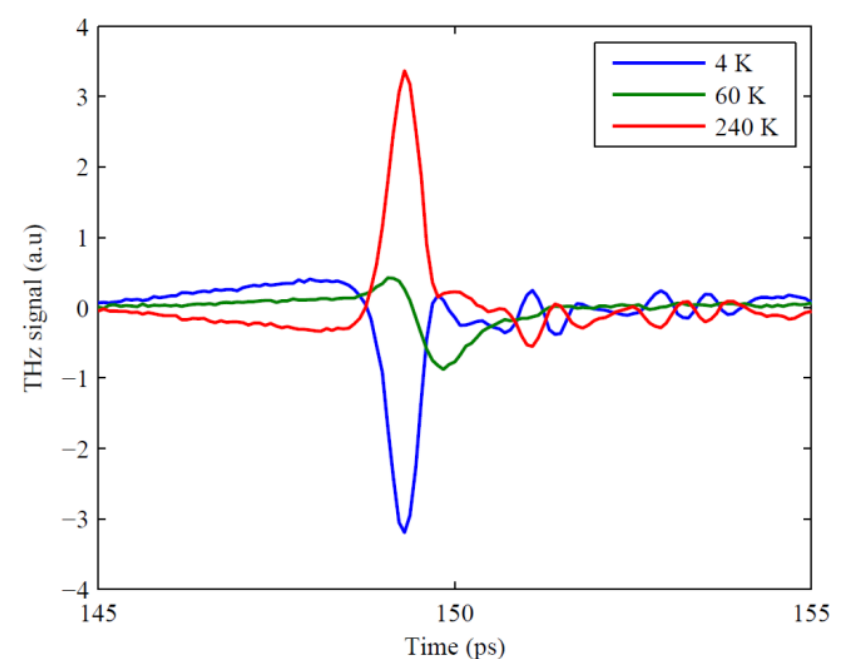

Fig. 4 Time domain scans for a single edge Au emitter at three different temperatures. The red line shows the THz TDS scan at $240 \mathrm{~K}$. As temperature is reduced the signal gradually decreases to the level shown by the green line at $60 \mathrm{~K}$. Below this temperature the polarity reverses and amplitude gradually increases to the large, inverted signal at $4 \mathrm{~K}$ shown by the blue line. completely applicable in the case of the DM emitters as there is also the Schottky effect on the surface fields from the presence of the metals. However, competing mechanisms would explain why the uninsulated $\mathrm{Au} / \mathrm{Pb}$ emitter exhibits a lower $\mathrm{THz}$ output than the insulated $\mathrm{Au} / \mathrm{Pb}$ emitter in the region where electron mobility is at its highest value.

Conclusion: The temperature dependent polarity inversion in DM and single edge metal - semiconductor interface emitters was investigated. DM emitters utilising different metal pairings and single metal emitters were cooled in a helium flow cryostat and their $\mathrm{THz}$ emission was measured over a range of temperatures. All emitters, except the $\mathrm{Au} / \mathrm{Al}$, including those with insulating layers between the metal and semiconductor, exhibited a flip in polarity of their $\mathrm{THz}$ emission between 50 and $100 \mathrm{~K}$. This shows the inversion is a trait intrinsic to the semiconductor and not influenced by the metallic contact on the surface. The only emitter to not show a polarity inversion is that fabricated with $\mathrm{Au} / \mathrm{Al}$ metal pairing. This is attributed to the similar skin depths of the metals at $\mathrm{THz}$ frequencies.

Further work would seek to work on a simulation of carrier dynamics of our emitter designs in order to understand and take advantage of these polarity flip effects [8].

(All data supporting this study are openly available from the University of Southampton repository at http://doi.org/10.5258/SOTON/D0447)

P. C. Gow and S. A. Berry (Optoelectronics Research Centre, University of Southampton, Southampton, UK)

D. McBryde and V. Apostolopoulos (Physics and Astronomy, University of Southampton, Southampton, UK)

E-mail: p.gow@soton.ac.uk

\section{References}

1. McBryde, D., Gow, P., Berry, S. A., Barnes, M. E., Aghajani, A., Apostolopoulos, V. 'Multiple double-metal bias-free terahertz emitters', Appl. Phys. Lett., 2014, 104, (20), pp. 201108, doi: 10.1063/1.4878739

2. Dember, H. 'Über eine photoelektronische Kraft in KupferoxydulKristallen', Z. Phys., 1931, 32, pp. 554.

3. Gow, P.C., McBryde, D., Berry, S.A., Barnes, M.E., Apostolopoulos, V. 'Characterisation of $\mathrm{THz}$ emission from double-metal-patterned gallium-arsenide multiple emitters', Elec. Lett., 2014, 50, (25), pp. 1966-1968, doi: 10.1049/el.2014.2751

4. McBryde, D., Barnes, M.E., Berry, S.A., Gow, P., Beere, H.E., Ritchie, D.A., Apostolopoulos, V. 'Fluence and polarisation dependence of GaAs based Lateral Photo-Dember terahertz emitters', Opt. Express, 2014, 22, (3), pp. 3234-3243, doi: 10.1364/OE.22.003234

5. Jin, Y., Ma, X. F., Wagoner, G. A., Alexander, M., Zhang, X. C. 'Anomalous optically generated $\mathrm{THz}$ beams from metal/GaAs interfaces', Appl. Phys. Lett., 1994, 65, (6), pp. 682-684, doi: $10.1063 / 1.112267$

6. Wolfe, C.M., 'Electron mobility in high-purity GaAs', J. Appl. Phys., 1970, 41, (7), p. 3088 , doi: $10.1063 / 1.1659368$

7. Nakajima, M., Hangyo, M., Ohta, M., Miyazaki, H. 'Polarity reversal of terahertz waves radiated from semi-insulating InP surfaces induced by temperature', Phys. Rev. B, 2003, 67, (19), pp. 195308, doi: 10.1103/PhysRevB.67.195308

8. Barnes, M.E., Daniell, G.J., Gow, P., Apostolopoulos, V. 'Simulation of terahertz generation from lateral diffusion currents in semiconductor devices', J Infrared Millim, Terahertz Waves, 2014, 35, (12), pp. 10301044, doi: 10.1007/s10762-014-0111-y 